\title{
Multi-objective optimization of rotary-wing aircrafts at the predesign stage
}

\author{
Florian Rigaud $^{1,2, a}$, Miguel Charlotte ${ }^{1}$, Christelle Kerdreux ${ }^{2}$ and \\ PIERRE MARECHAL ${ }^{3}$ \\ 1 Université de Toulouse, Institut Clément Ader, Institut Supérieur de l'Aéronautique et de l'espace, France \\ 2 Eurocopter, 13725 Marignane, France \\ 3 Université de Toulouse, Institut Supérieur de l'Aéronautique et de l'espace, France
}

Received 16 January 2014, Accepted 2 April 2014

\begin{abstract}
Subsystems of rotary-wing aircrafts, such as helicopters for instance, are strongly interrelated due to their intrinsic specificities. Convergence to a feasible design is then not ensured and implies an iterative process. Moreover, rotorcrafts must cover a much wider range of missions than their fixed-wing counterparts. For those reasons a correctly sized rotorcraft is difficult to obtain and finding the best design for a defined set of missions needs numerous iterations. This article presents the application of a multi-objective optimization approach from the predesign stage. The standard predesign approach has been reformulated to highlight sizing constraints and three strategies are then proposed to solve those constraints: the first one is the basic transcription of the standard predesign approach; the second one leaves the problem solving to the genetic optimizer through penalization; the third one is a hybrid of both previous methods based on a constraint repairing approach. Those strategies also involved the adaptation of the helicopter modelling. Here, the focus is on two components of that new model: namely the main rotor polar and the weight assessment model.
\end{abstract}

Key words: Helicopter / optimization / predesign / sizing / penalization

\section{Introduction}

Vertical take-off and landing aircrafts (VTOL) are, among all transportation means, from those for which the conception phase involves the highest number of compromises. In order to perform flights in hover as well as in high speed cruise, they have to meet technical and environmental requirements. In practice, those aspects of their missions appear to imply antagonistic constraints. A rotorcraft cannot entirely satisfy each of them. As an example, increasing the main rotor diameter, in order to improve the hovering performance, implies an increase of both the empty weight and the drag brought to high speed cruise. Moreover, the blade tip speed being limited by acoustic considerations, this increase of diameter implies a decrease of the rotor rotation speed. Transmitted torques will then increase also implying higher anti-torque trust at the aft rotor, both leading to an increase of the empty weight. This example illustrates the main problematic in rotorcraft conception: trade-offs are difficult to obtain since all subsystems are remarkably interrelated.

\footnotetext{
${ }^{a}$ Corresponding author: florian.rigaud@outlook.com
}

A modification applied to one of them (e.g. the main rotor) may indeed affect most of the others (e.g. the drive system, the engines and the tail rotor). The impact of any modification has then to be considered in the frame of the entire aircraft and its missions.

One of the key steps encountered during the development of a new rotorcraft is the predesign phase. Indeed, the great majority of the final cost will be a consequence of the choices done during this phase [1]. Moreover, once in development phase, any modification made to those choices will be expensive. It is therefore essential to ensure that the designed aircraft will be able to perform of all the missions it is dedicated to.

The requirements to set up a rotorcraft definition associated to these missions are enclosed in a specification document. They can, for example, be expressed as being able to take-off at full weight at 5000 feet or to take 8 passengers to a distance of 120 nautical miles and bring them back. The first goal is referred to as a flight case while the second is a mission. As a consequence, the transcription of such requirements mainly leads to design constraints. Matching all specifications is then in itself a difficult task. 


\section{Nomenclature}

\begin{tabular}{|c|c|c|c|}
\hline Notation & Denomination & Notation & Denomination \\
\hline$b$ & Blade number & $\bar{p}$ & Size of a flight condition vector \\
\hline$c$ & Blade chord, m & $P_{\mathrm{I}}$ & Induced power, $\mathrm{W}$ \\
\hline$C_{x \mathrm{c}}$ & Profile drag compressibility correction & $P_{\mathrm{P}}$ & Profile power, W \\
\hline$C_{x i}$ & Basic profile drag coefficient & $P_{\mathrm{F}}$ & Fuselage power, W \\
\hline$C_{x \mathrm{p}}$ & Total mean profile drag coefficient & $P_{\mathrm{C}}$ & Climb power, W \\
\hline$C_{x S}$ & Fuselage drag reference area, $\mathrm{m}^{2}$ & $P_{\mathrm{av}}$ & Available power, $\mathrm{W}$ \\
\hline$C_{z \max }$ & Maximum admissible lift coefficient & $R$ & Main rotor radius, $\mathrm{m}$ \\
\hline$C_{z \mathrm{~m}}$ & Mean lift coefficient & $R_{e}$ & Reynolds number \\
\hline$D_{1}, D_{2}, D_{3}$ & Penalization coefficients & $R_{S}$ & Gas constant, J.kg ${ }^{-1} \cdot \mathrm{K}^{-1}$ \\
\hline$e$ & Rotor eccentricity, m & $s$ & Number of objective functions \\
\hline$E$ & Search space & $S$ & Feasible aircraft space \\
\hline$f_{i}$ & $i$ th objective functions & $T$ & Outside air temperature, $\mathrm{K}$ \\
\hline$F_{n}$ & Rotor thrust, $\mathrm{N}$ & $T$ & Blade mean thickness, $\mathrm{m}$ \\
\hline$g$ & $\mathrm{~g}$-force acceleration, $\mathrm{m} \cdot \mathrm{s}^{-2}$ & $U$ & Main rotor tip speed, $\mathrm{m} \cdot \mathrm{s}^{-1}$ \\
\hline$h_{i}$ & $i^{\text {th }}$ limitation functions & $V_{x}$ & Horizontal speed, $\mathrm{m} . \mathrm{s}^{-1}$ \\
\hline$J$ & Main rotor moment of inertia, $\mathrm{kg} \cdot \mathrm{m}^{2}$ & $V_{z}$ & Vertical speed, $\mathrm{m} . \mathrm{s}^{-1}$ \\
\hline$k_{\mathrm{b}}, k_{\mathrm{b}}^{\prime}$ & Coefficients for the blade mass assessment & $W_{1}$ & Main rotor required power, $\mathrm{W}$ \\
\hline$k_{\mathrm{c}}, k_{\mathrm{c}}^{\prime}$ & Coefficients for the control mass assessment & $W_{2}$ & Tail rotor required power, $\mathrm{W}$ \\
\hline$k_{\mathrm{e}}, k_{\mathrm{e}}^{\prime}, k_{\mathrm{e}}^{\prime \prime}$ & Coefficients for the engine mass assessment & $W_{e}$ & Engine available power, $\mathrm{W}$ \\
\hline$k_{\mathrm{h}}, k_{\mathrm{h}}^{\prime}$ & Coefficients for the hub mass assessment & $W_{\mathrm{rp}}$ & Total required power, $\mathrm{W}$ \\
\hline$k_{\mathrm{m}}, k_{\mathrm{m}}^{\prime}$ & Coefficients for the mast mass assessment & $X$ & Vector of the design variables \\
\hline$k_{\mathrm{p}}, k_{\mathrm{p}}^{\prime}, k_{\mathrm{p}}^{\prime \prime}$ & Coefficients for the profile drag polar & $X^{\prime}$ & Vector of both design variables and limit value \\
\hline$k_{\mathrm{p}}^{*}, k_{\mathrm{p}}^{* *}$ & & $z_{1}, z_{2}, z_{3}$ & Maximum admissible torques at main roto $\mathrm{r}(1)$, \\
\hline$K$ & Limit value space & & tail rotor (2) and main gear box input (3), N.m \\
\hline$m$ & Number of limitations considered & $z_{4}$ & Maximum take-off weight, $\mathrm{kg}$ \\
\hline$M$ & Rotorcraft total mass, $\mathrm{kg}$ & $z_{5}$ & Fuel capacity, kg \\
\hline$M_{\mathrm{a}}$ & Mach number at $70 \%$ of the radius & $z_{6}$ & Engine nominal available power, $\mathrm{W}$ \\
\hline$M_{\mathrm{b}}$ & Blades mass, kg & $Z$ & Vector of the limit values \\
\hline$M_{\mathrm{c}}$ & Rotor controls mass, $\mathrm{kg}$ & $\alpha$ & Blade angle of attack, rad \\
\hline$M_{\mathrm{cs}}$ & Blade current section mass, $\mathrm{kg}$ & $\gamma$ & Heat capacity ratio \\
\hline$M_{\mathrm{dd}}$ & Drag divergence Mach number & $\rho$ & Density of air, $\mathrm{kg} \cdot \mathrm{m}^{-3}$ \\
\hline$M_{\mathrm{e}}$ & Rotorcraft empty weight, kg & $\omega$ & Main rotor rotation speed, $\operatorname{rad} . \mathrm{s}^{-1}$ \\
\hline$M_{E E}$ & Empty weight equipped, $\mathrm{kg}$ & $\omega_{2}$ & Tail rotor rotation speed, rad.s ${ }^{-1}$ \\
\hline$M_{\text {fuel }}$ & Fuel uplift weight, kg & $\omega_{\mathrm{GB}}$ & Main gearbox reference speed, ${\mathrm{rad} . \mathrm{s}^{-1}}^{-1}$ \\
\hline$M_{\mathrm{g}}$ & Maximum gross weight, $\mathrm{kg}$ & $\eta$ & Induced efficiency \\
\hline$M_{\mathrm{h}}$ & Rotor hub mass, kg & $\nu$ & Kinematic viscosity, $\mathrm{m}^{2} \cdot \mathrm{s}^{-1}$ \\
\hline$M_{\mathrm{m}}$ & Rotor mast mass, $\mathrm{kg}$ & $\Omega$ & Set of flight conditions \\
\hline
\end{tabular}

Since the predesign stage is the earliest phase, the realm of possibility is still widely open and every configuration should be considered. This is why optimizing the design is often considered as secondary. One should keep in mind, however, that the aircraft must also stand out from its competitors, which motivates resorting to optimization as soon as possible.

Most of the helicopter manufacturers and several laboratories and universities are currently working on tools dedicated to helicopter predesign. A brief overview of the main projects and their scope are presented in Table 1.

The CREATION code (Concepts of Rotorcraft Enhanced Assessment Through Integrated Optimization Network) is developed by the ONERA since 2010 [2]. It includes several performance models from the basic power balance assessment to the blade element theory,
Table 1. Existing helicopter design tools.

\begin{tabular}{|c|c|c|c|c|}
\hline Name & Organism & Optim & $\begin{array}{l}\text { Weight } \\
\text { models }\end{array}$ & $\begin{array}{l}\text { Perfos } \\
\text { models }\end{array}$ \\
\hline NDARC & NASA & $\mathrm{NO}$ & YES & $\begin{array}{c}\text { Basic to } \\
\text { advanced }\end{array}$ \\
\hline CREATION & ONERA & YES & $\begin{array}{l}\text { YES } \\
\text { (basic) }\end{array}$ & $\begin{array}{l}\text { Basic to } \\
\text { advanced }\end{array}$ \\
\hline SPEARS & NLR & $\mathrm{NO}$ & YES & Basic \\
\hline CRDS & TUM & YES & $\mathrm{NO}$ & Basic \\
\hline
\end{tabular}

but has no weight model so far [3]. It then cannot perform sizing calculations. SPEARS (SPEcification Analysis of Rotorcraft) is developed by the national aerospace laboratory of the Netherlands [4]. It mainly aims at being a performance tool and does not include sizing loops yet. 
The NASA code NDARC (NASA Design and Analysis of Rotorcraft) is able to cover a great variety of models [5]. Even if it is one of the most advanced performance estimation model, it does not include optimization capabilities yet. The Technische Universität Munchen (TUM) proposes advanced constraint management and enhanced optimization with their CRDS code (Compound Rotorcraft Design Suite). However, performance and weight models are strongly simplified [6].

Currently, there is then no computational software code able to perform both a full sizing and a complete multi-objective optimization of a future helicopter development, at predesign stage. We propose both a generic integrated helicopter model and an optimization strategy bringing a solution to the complex multi-objective and multi-disciplinary problem of the helicopter predesign at a reduced time cost.

The proposed code allows for drastic reduction of the time needed to get to a final design. At the same time, it provides all the information the user needs to understand the problem underlying structure and thus to choose among the set of compromises. Finally this optimization tool is robust and usable by someone untrained to optimization.

For the purpose of this study, the optimization strategy is applied to a toy model. It is composed of simplified models created from bibliography and well known results reflecting most of the behaviour of the more sophisticated ones.

The paper is organized as follows. In Section 2, we describe the model and state the problem. In Section 3, we specify all constraints accounted for in our toy problem, in view of the statement of the optimization problem. Section 4 is devoted to the numerical results and their interpretation.

\section{Problematic and modelling approach}

\subsection{Problem description}

The helicopter sizing problem consisting in finding a rotorcraft capable of meeting all the requirements of the specification can be abstractly stated as follows. The aircraft is defined as a set of design variables, forming a real vector $X$, and a set of sizing limit values, gathered in another real vector $Z$. The requirements are defined in terms of flight cases to hold and missions to be performed. Because of the required level of fidelity, this work chooses not to simulate the helicopter's dynamic and transient phases, both being costly in terms of computation time. Missions can then be discretised into static time steps. This means that both flight cases and missions can be reduced as a set of flight conditions forming a discrete space $\Omega$ of vectors of $\mathbb{R}^{p}$.

The sizing problem consists then in finding one ordered pair $(X, Z)$ in $E \times K$, where $E$ and $K$ denote the search space and the sizing variable space, respectively. The set of solutions to the problem is given by

$$
S:=\left\{X \in E, Z \in K \mid \forall i \in \llbracket 1 ; m \rrbracket, \quad h_{i}(X, Z, \Omega) \leqslant 0\right\}
$$

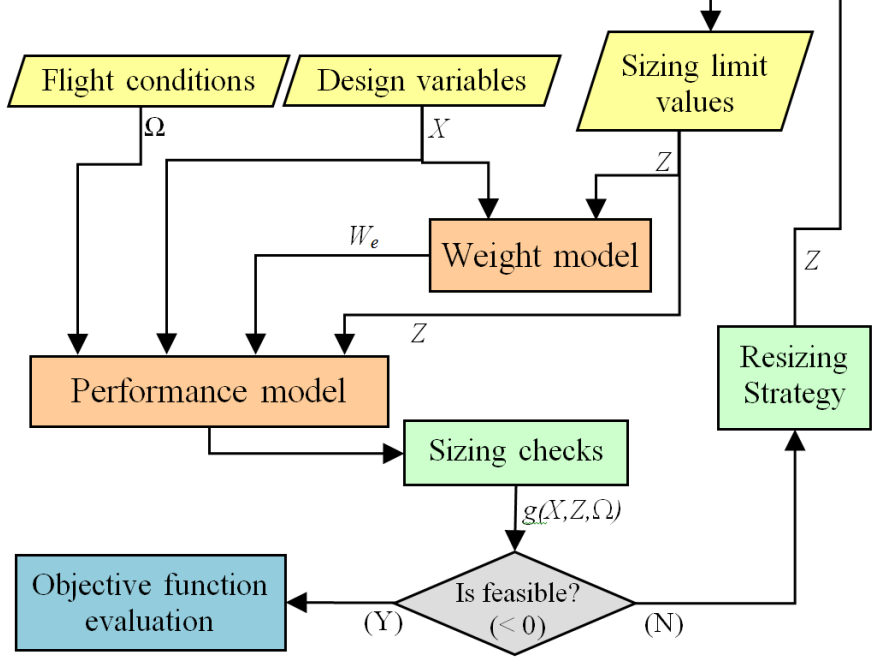

Fig. 1. Model flowchart.

Here, $h_{i}$ is the $i$ th considered limitation as described in Section 3.1. Although it is customary to only focus on this sizing problem (i.e. finding one element of $S$ with no optimality consideration), we are interested here in selecting, among all members of $S$, a set of solutions optimizing several criteria.

\subsection{Aircraft behaviour modelling}

The database structure is thought to cover any rotorcraft configuration from the classic main-rotor/tailrotor helicopter to quad-rotors compounds through tandem configurations for example. The approach chosen to get this flexibility defines the rotorcraft as the sum of several generic components. These components can be rotors, lifting surfaces or engines. This results in at most 70 degrees of freedom for a compound aircraft. In practice, for a standard rotorcraft, about 8 variables are sufficient. Three modules are implemented: a performance model, an engine model and a weight model.

Both the weight and the performance models lead to a set of flight data. Then, the above mentioned constraints are tested for the obtained data, so as to determine whether the aircraft is correctly sized. A very simplified flow chart of the complete model is presented in Figure 1.

The performance model simulates the helicopter behaviour according to the flight conditions. The calculated data are, among others, the required power, the fuel consumption, the rotor lift, etc. This model is presented in Section 2.3. The weight model assesses the empty weight of the aircraft according to geometrical data from the design variables and the limitations; it is presented in Section 2.4 .

\subsection{Performance model}

In the frame of this study, a simplified power balance model (as presented in [7]) has been used. The required 
power is assessed for each rotor and a drive system model sums it into a total required power, $W_{\text {req }}$. Details of this model, of the engine consumption and the global mission simulation are not given in this paper for the sake of simplicity. Only the main rotor assessment is described. Four power needs are identified at the main rotor:

- the induced power $P_{\mathrm{I}}$, which creates the lift;

- the profile power $P_{\mathrm{P}}$, which compensates for the blades drag;

- the fuselage power $P_{\mathrm{F}}$, which compensates for the body drag;

- the climb power $P_{\mathrm{C}}$.

Using the Froude theory and the assumption of a horizontal rotor disc described in [8], the induced power can be assessed as follow:

$$
P_{\mathrm{I}}=\frac{1}{\eta} F_{n} \sqrt{\sqrt{\left(\frac{V_{x}^{2}}{2}\right)^{2}+\left(\frac{F_{n}}{2 \rho \pi R^{2}}\right)^{2}}-\frac{V_{x}^{2}}{2}}
$$

Here, $\frac{1}{\eta}$ is a correction factor set to $1.1, F_{n}$ is the total rotor thrust, $V_{x}$ is the rotorcraft horizontal speed, $\rho$ is the density of air and $R$ is the main rotor radius.

The total rotor power can then be expressed as

$$
W_{1}=P_{\mathrm{I}} \underbrace{+\frac{1}{8} \rho b c R^{4} \omega^{3} C_{x \mathrm{p}}}_{P_{\mathrm{p}}} \underbrace{+\frac{1}{2} \rho C_{x S} V_{x}^{3}}_{P_{\mathrm{f}}}+\underbrace{M g V_{z}}_{P_{\mathrm{c}}}
$$

Here, $C_{x \mathrm{p}}$ is the mean profile drag coefficient, $b$ is the number of blades, $c$ is the blade chord, $\omega$ is the rotation speed, $C_{x S}$ is the fuselage drag reference area and $M$ is the rotorcraft total mass. This coefficient is usually assessed from the rotor polar of a state of the art rotor definition. It is then a fixed polar that does not account for modification of the rotor radius or the rotor rotation speed for example. In the frame of an integrated sizing loop, the rotor characteristics are part of the design variables. It is then required to be able to create the rotor polar from the rotor characteristics. The following paragraph presents the predictive rotor polar model produced for this purpose in hovering conditions. A similar approach has been applied to develop the polar in cruise conditions.

\subsubsection{Predictive polar for the profile drag in hover}

The proposed model is built as the sum of an initial basic polar and different corrections accounting for the influence of specific effects. These corrections account for

- the Reynolds number;

- the compressibility;

- the thrust effect.

For obvious confidentiality issues, the method presented in this paragraph is applied to a well-known NACA0012 airfoil instead of a new generation airfoil.

Since blades are rotating, the Reynolds number varies along the radius. As shown in [9] the maximum induced

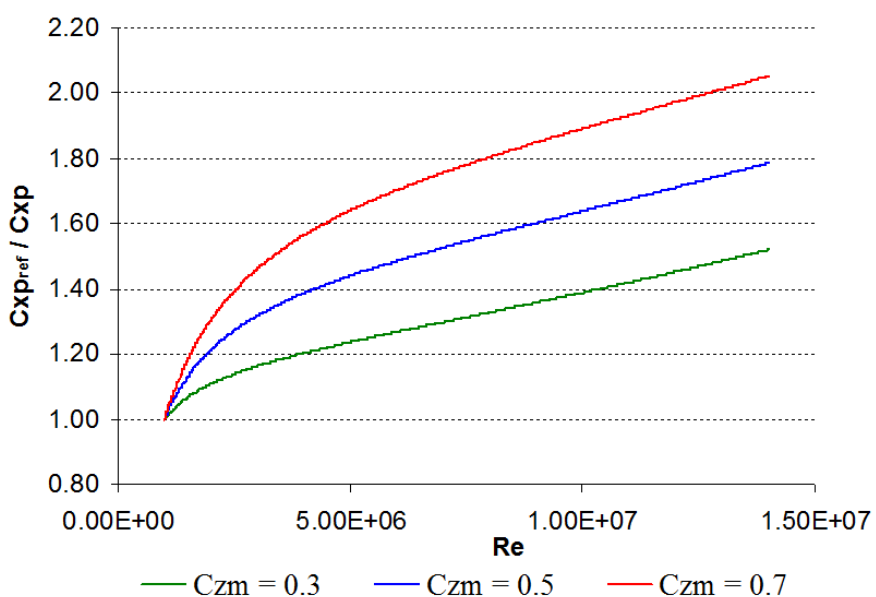

Fig. 2. Evolution of the Reynolds correction.

speed, hence the maximum lift, are produced at a distance around $0.7 \times R$ of the rotor center. For this reason, the reference Reynolds number [10] is chosen as

$$
R_{e}=\frac{0.7 c R \omega}{\nu}
$$

Here, $\nu$ is the kinematic viscosity of air. The first step of the polar construction is to produce a drag coefficient from the bi-dimensional profile data. The proposed formulation is the following:

$$
C_{x i}=C_{x 0}\left(R_{e}\right)+k\left(R_{e}\right) C_{z \mathrm{~m}}^{2}
$$

Here, $C x_{0}$ and $k$ depend on the Reynolds number and $C_{\mathrm{zm}}$ is the rotor mean lift coefficient. Using the NACA0012 abacus presented in [10] and considerations from [11], they can be expressed as

$$
C_{x 0}\left(R_{e}\right)=k_{\mathrm{p}}-k_{\mathrm{p}}^{\prime} R_{e}
$$

and

$$
k\left(R_{e}\right)=k_{\mathrm{p}}^{\prime \prime}+\frac{k_{\mathrm{p}}^{*}}{R_{e}}
$$

Here, $k_{\mathrm{p}}, k_{\mathrm{p}}^{\prime}, k_{\mathrm{p}}^{\prime \prime}$ and $k_{\mathrm{p}}^{*}$ are positive real coefficients. The selected coefficients lead to the evolution with the Reynolds number presented in Figure 2.

The second effect considered is the compressibility. This appears at the blade tip and is mainly driven by the Mach number. For rotary wings the Mach number [10] is defined at the blade tip by:

$$
M_{\mathrm{a}}=\frac{U}{\sqrt{\gamma R_{s} T}}
$$

Here, $\gamma$ is the heat capacity ratio, $R_{s}$ is the gas constant and $T$ is the outside air temperature. The correction will be applied when the drag divergence Mach number, $M_{\mathrm{dd}}$, is reached. From the data provided by [10], $M_{\mathrm{dd}}$ can be considered as a function of the angle of attack as shown in Figure 3.

It can be derived from [12] that, for the range of acceptable blade aspect ratio, the mean lift coefficient 


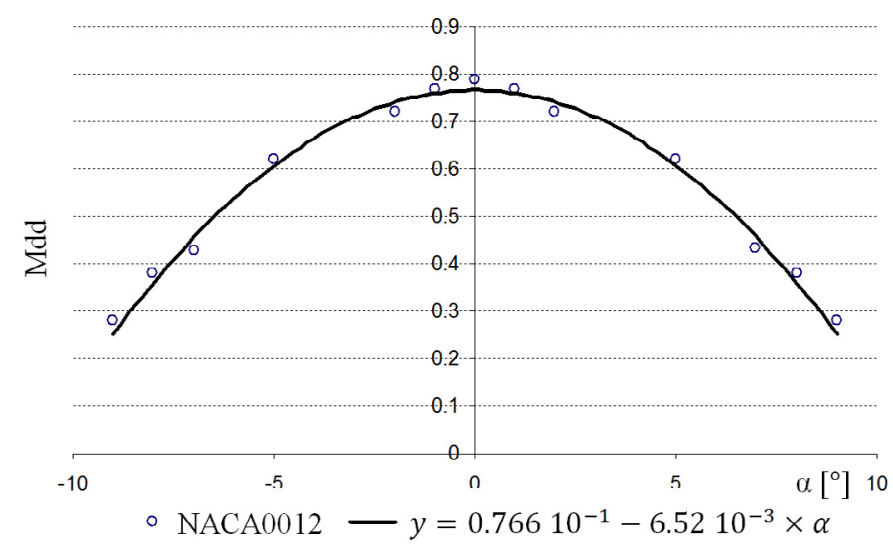

Fig. 3. Drag divergence Mach number (NACA0012).

and the angle of attack vary according to the formula $C_{z \mathrm{~m}}=5.7 \propto$, with $\propto$ in radians. Hence,

$$
M_{\mathrm{dd}}=0.76610^{-1}-6.5210^{-1} C_{z \mathrm{~m}}
$$

The compressibility correction is then considered to be a quadratic function of the distance between the actual Mach and the drag divergence Mach:

$$
C_{x \mathrm{c}}=k_{\mathrm{p}}^{* *}\left(M_{\mathrm{a}}-M_{\mathrm{dd}}\right)^{2}
$$

Here, $k_{\mathrm{p}}^{* *}$ is a positive real coefficient and is assessed from whirl tower testing.

The assessed complete mean profile drag coefficient is then

$$
C_{x \mathrm{p}}=C_{x 0}\left(R_{e}\right)+k\left(R_{e}\right) C_{z \mathrm{~m}}^{2}+k_{\mathrm{p}}^{* *}\left(M_{\mathrm{a}}-M_{\mathrm{dd}}\right)^{2}
$$

The presented approach has been applied to Eurocopter profiles and compared to whirl tower measurements. Figure 4 below shows the relative error between the assessed polar and the measured one for a range of three different blade tip Mach numbers.

\subsection{Modelling of the aircraft empty weight}

The second model brought into focus in this study is the mass assessment model. Mass assessment is one of the key elements in rotorcraft sizing. The empty weight reached has indeed a huge impact on the final capabilities of the developed aircraft. For example, a medium aircraft of the 5 tons class can be estimated to be around 3 tons of empty weight. An underestimation of $5 \%$ on each subsystem would lead to a difference of 150 kilograms between the project and the achieved empty weights, which corresponds to the mass of more than one passenger. It is then important to be able to precisely assess the weight of the aircraft according to few key parameters.

The empty weight is defined as the weight of the helicopter without any crew, passenger, freight and fuel. It is a function of the design variables and the sizing limit values. The main gear box mass for example will depend,

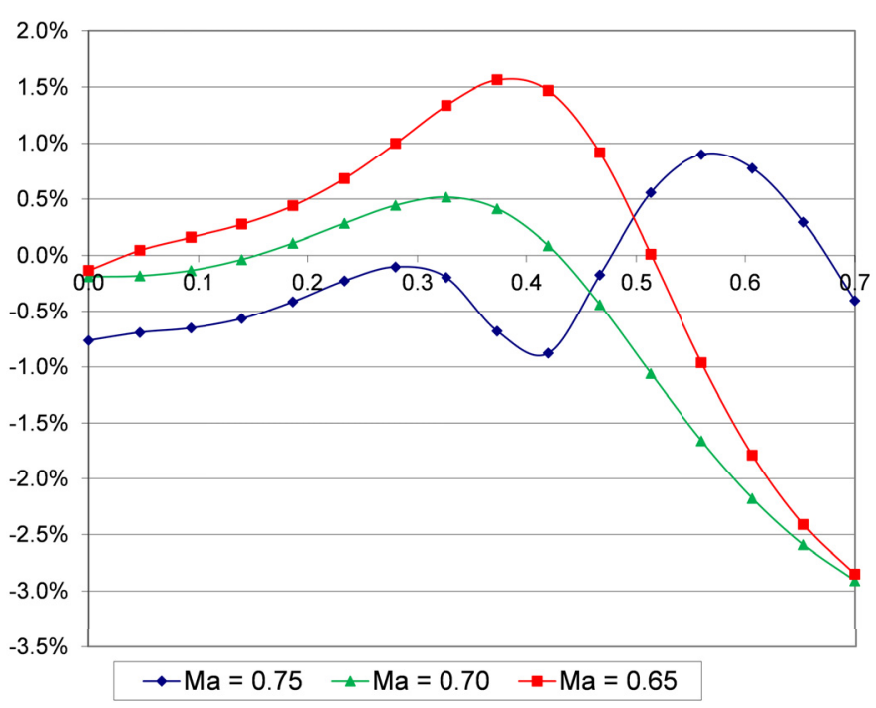

Fig. 4. Predictive polar against whirl tower tests: relative errors.

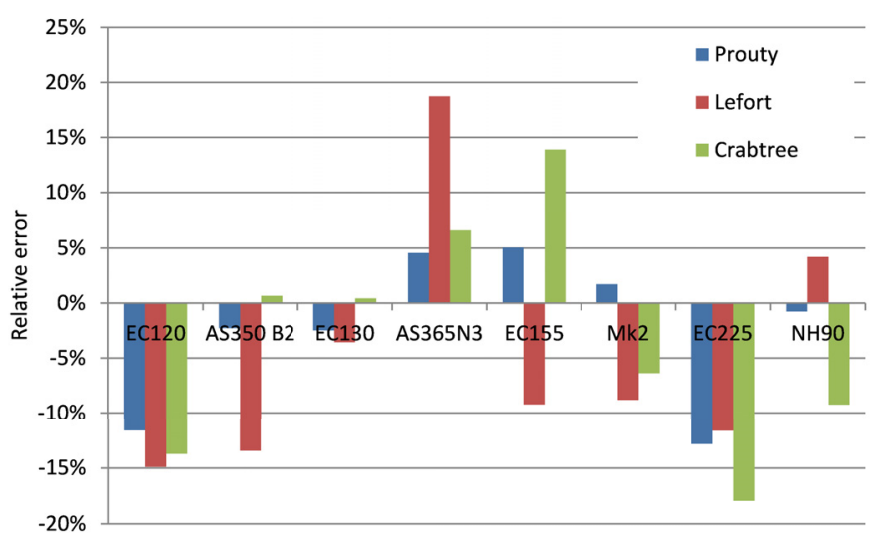

Fig. 5. Relative errors for the main rotor weight estimation.

among others, on the rotor rotation speed and the maximum torque it can transfer [13].

Because each system is designed by a specific set of variables, the aircraft has been divided into the following 12 systems:

$\begin{array}{ccc}\text { Main rotor } & \text { Landing gear } & \text { Hydraulic power } \\ \text { Structure } & \text { Electrical power } & \text { Main rotor drive } \\ \text { Tail rotor } & \text { Power plant } & \text { Tail rotor drive } \\ \text { Avionics } & \text { Fuel system } & \text { Equipment }\end{array}$

The empty weight is then assessed as the sum of the masses of all of the aircraft systems as follow.

$$
M_{\mathrm{e}}=\sum_{i=1}^{12} M_{\mathrm{i}}
$$

where $M_{\mathrm{i}}$ is the weight of the $i$ th system.

Weight estimation laws have been produced for each system of the rotorcraft. The following paragraphs present the approach chosen to produce the main rotor one. 


\subsubsection{Main rotor mass estimation}

The rotor is composed of a rotor mast, a rotor central hub, the sleeves, the dampers, the blades, the rods and the swash plate. Different technologies can be used to connect the flapping masses to the hub. Oldest ones used bearings on each articulation and are referred to as articulated rotors. The existing laws were created for rotors using this technology. Recent helicopters use a different one where bearings have been replaced by flexible materials such as elastomer. This technology, called semi-rigid, drastically reduces the number of parts required, which increases the reliability. Existing laws are then not applicable to this new technology and it is necessary to produce specific weight estimation models.

The chosen approach aims at transposing as faithfully as possible the design as it is actually made in the design office. The rotor is subdivided according to the sizing load applied to each element.

\begin{tabular}{cc}
\hline Element & Sizing loads \\
\hline Blades & $\begin{array}{c}\text { Distributed loads of lift and } \\
\text { centrifugal force }\end{array}$ \\
Hub & $\begin{array}{c}\text { Centrifugal load generated by } \\
\text { the blades }\end{array}$ \\
Mast & $\begin{array}{c}\text { Mast torque longitudinal and } \\
\text { lateral moments } \\
\text { Controls }\end{array}$ \\
\hline
\end{tabular}

The weight of the blades, hub, mast and controls will have the subscripts $b, h, m$ and $c$, respectively. The subscript $b h$, for example, means that the considered weight refers to the sum of the blade and hub weights. Some weight estimation approaches can be found in the literature and are presented above. Three of them have been selected as the most interesting for a predesign stage application. The first one was presented by Crabtree in [14]:

$$
M_{\mathrm{hmcb}}=3.45 \times 10^{-4}\left(R \mathrm{cb} U^{2}(t+0.21)\right)^{0.89}
$$

Here, $t$ denotes the mean thickness of the blades.

The following laws were established by Lefort in [15]:

$$
\begin{aligned}
M_{\mathrm{b}} & =\frac{841}{U^{2} R^{2}}\left(\frac{z_{4}^{5}}{b^{2}}\right)^{\frac{1}{3}} \\
M_{\mathrm{hmc}} & =23.4\left(M_{\mathrm{b}} R \omega^{2} z_{1} e^{1.82} b^{1.5} \times 10^{-9}\right)^{0.358}
\end{aligned}
$$

Here, $e$ is the rotor eccentricity, $z_{4}$ is the maximum takeoff weight and $z_{1}$ is the maximum admissible torque at main rotor mast.

The last example of weight estimation comes from RW Prouty's book [16]:

$$
\begin{aligned}
M_{\mathrm{b}} & =0.026 \times b^{0.66} c R^{1.3} U^{0.67} \\
M_{\mathrm{hmc}} & =3.7 \times 10^{-4} \times b^{0.28} R^{1.5} U^{0.43}\left(0.67 M_{\mathrm{b}} \frac{g J}{R^{2}}\right)^{0.55}
\end{aligned}
$$

where $J$ is the moment of inertia of the complete rotor.

Those models do not present satisfying results over the considered range of helicopters. The used parameters are however a good starting point for the development of a new model, as shown in the following paragraphs.

\section{The main rotor hub}

The following parameters are considered to be predominant in the design of the rotor hub:

\begin{tabular}{cc}
\hline Geometrical data & Loads and moments \\
\hline Blade number & Centrifugal force \\
Radius & Maximum lift \\
Eccentricity & Flapping torque \\
Length of the sleeves & \\
\hline
\end{tabular}

Maximum lift can be quickly ruled out of the list of key parameters. Indeed it is negligible compared to the centrifugal forces. A Eurocopter Ecureuil for example is a three bladed helicopter with a maximum gross weight of about 2.2 tons. Its maximum lift will then be around $7 \mathrm{kN}$ per blade, while a radius of $5.3 \mathrm{~m}$, a peripheral speed of $220 \mathrm{~m} . \mathrm{s}^{-1}$ and a blade weight around $35 \mathrm{~kg}$ provide a centrifugal load of $80 \mathrm{kN}$ at each sleeve. At the predesign stage, the centrifugal load generated by one blade can be computed as:

$$
F_{c}=R \omega^{2} M_{\mathrm{b}}
$$

Furthermore, studies on the geometrical data statistics have shown that satisfactory levels of correlation are reached for the rotor radius only.

The proposed estimation is then chosen as the following:

$$
M_{\mathrm{h}}=k_{\mathrm{h}} \omega^{2} R^{2} M_{\mathrm{b}}+k_{\mathrm{h}}^{\prime}
$$

with $k_{\mathrm{h}}$ and $k_{\mathrm{h}}^{\prime}$ positive real coefficients assessed from the Eurocopter weight database.

\section{The main rotor mast}

The following parameters are considered to be predominant in the design of the rotor mast:

\begin{tabular}{cc}
\hline Geometrical data & Loads and moments \\
\hline Mast length & Flapping torque \\
& Max transmitted torque \\
\hline
\end{tabular}

The geometric data of the mast length are not currently considered as accessible at the predesign stage. From the weight database, the maximum transmitted torque appears to be the main factor in the mast sizing. The proposed formulation is then

$$
M_{\mathrm{m}}=k_{\mathrm{m}} z_{1}^{k_{\mathrm{m}}^{\prime}}
$$

with $k_{\mathrm{m}}$ and $k_{\mathrm{m}}^{\prime}$ positive real coefficients assessed from the Eurocopter weight database. 


\section{The main rotor controls}

Controls are designed according to the maximum control loads. Estimating those loads implies advanced dynamic calculations and then requires a complete definition of the rotor. It is therefore necessary to directly use the rotor geometrical data. A statistical study has shown that those loads are mainly driven by the total blade surface. The database analysis showed that the following formulation provides good results on the available helicopter range.

$$
M_{\mathrm{c}}=k_{\mathrm{c}} b^{2} c^{2} R^{2}+k_{\mathrm{c}}^{\prime}
$$

with $k_{\mathrm{c}}$ and $k_{\mathrm{c}}^{\prime}$ positive real coefficients assessed from the Eurocopter weight database.

\section{The main rotor blades}

Blades are divided into three sections; the root (from the rotor centre to $0.27 R$, the current section (from $0.27 R$ to $0.95 R$ ) and the blade tip. It appears that the blade tip could be considered as a current section portion of $0.3 R$. The linear density turns out to be constant in the current section and proportional to the chord. The linear density of the current section can then be assessed as $k_{\mathrm{b}} c$.

The blade root has the same function as the hub sleeves; holding masses subject to centrifugal loads. The proposed formulation is then similar to the weight estimation law of the hub, hence

$$
M_{\mathrm{root}}=k_{\mathrm{b}}^{\prime} M_{\mathrm{cs}} R^{2} \omega^{2}
$$

Here $M_{\mathrm{cs}}$ the current section and blade tip weight. The total weight of the blades is then:

$$
M_{\mathrm{b}}=b(0.98-0.27) R k_{\mathrm{b}} c\left(1+k_{\mathrm{b}}^{\prime} R^{2} \omega^{2}\right)
$$

with $k_{\mathrm{b}}$ and $k_{\mathrm{b}}^{\prime}$ positive real coefficients assessed from the Eurocopter weight database.

Figure 6 shows the relative errors for each aircraft of the weight database. The maximum encountered errors remain in the acceptable range defined above of $\pm 5 \%$.

To summarize, several new models have been developed in order to be integrated in the sizing process. As shown by both the predictive polar and the rotor weight assessment the major issue in the predesign model development consists in getting to a compromise between model fidelity and accessible variables.

\section{Sizing limitations: description and solving}

\subsection{Definition of the limitations used}

The design of a new aircraft is subject to strong limitations such as the engine available power and the gearboxes admissible torques. Most of those constraints are upper bounds which impact the empty weight of the aircraft and hence its performances. Minimizing those values

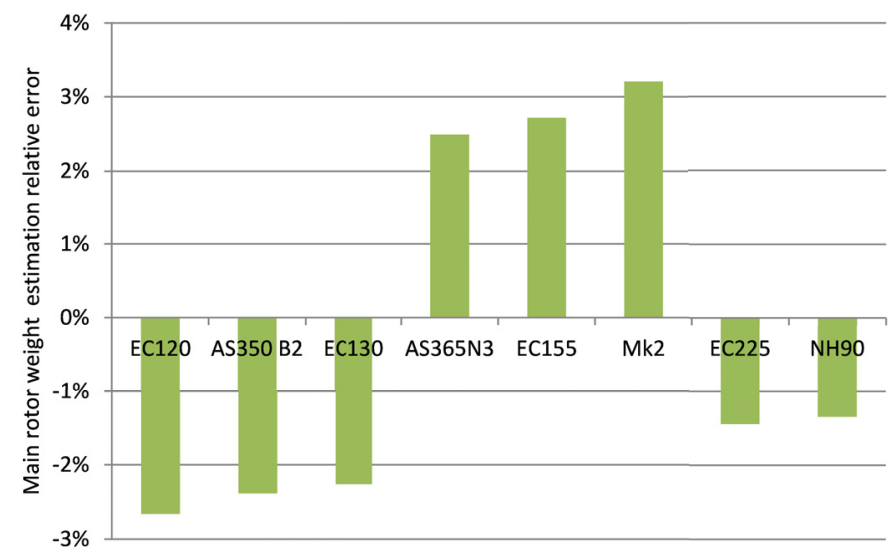

Fig. 6. Main rotor weight estimation error.

is then an iterative process. Moreover, since rotary-wings parameters are strongly interrelated, the resulting loop can call for an important number of iterations or lead to technically unrealistic aircrafts.

A specifically adapted sizing strategy is then required. For the purpose of this study, five of the most usual limitations are considered. They are related to the rotor, the drive system, the structure, the fuel system and the engines.

The rotor

A rotor cannot generate an infinite amount of thrust and lift. There is a maximum lift coefficient that can't be exceeded for aerodynamic reasons such as stall effects. This results in the following limitation:

$$
\max _{\Omega}\left(\frac{6 F_{n}}{\rho b c R^{3} \omega^{2}}\right)-C_{z \max } \leqslant 0 .
$$

The coefficient $C_{z \max }$ is the maximum admissible lift coefficient. It is linked to the rotor technology and will be chosen equal to 0.6 for the study. This limitation has an impact on the rotorcraft geometry $(b, c, R)$.

\section{The drive system}

The drive system aims at distributing the power from the producer (the engines) to the consumers (the rotors). The torque that can be transmitted is however limited by admissible values. Those limit values are defined at 3 different points: the main rotor mast, the tail rotor mast and the main gearbox addition stage, they are denoted $z_{1}$, $z_{2}$ and $z_{3}$ respectively. We obtain the constraints:

$$
\begin{aligned}
& \max _{\Omega}\left(\frac{W_{1}}{\omega}\right)-z_{1} \leqslant 0, \quad \max _{\Omega}\left(\frac{W_{2}}{\omega_{2}}\right)-z_{2} \leqslant 0, \\
& \max _{\Omega}\left(\frac{W_{r q}}{\omega_{G B}}\right)-z_{3} \leqslant 0
\end{aligned}
$$

These limitations also depend on the aircraft geometry. The main difference with the rotor limitation is due to the 
fact that $z_{1}, z_{2}$ and $z_{3}$ are limit values and then unknown. They should be defined during the sizing phase. Since they all impact the empty weight and therefore $W_{1}, W_{2}$ and $W_{G B}$, their assessment leads to an iterative process.

\section{The structure}

The structure sizing case is linked to crash certification requirements. Because the crash loads are linked to the aircraft gross weight, certificating an aircraft imposes the definition of a maximum gross weight, $z_{4}$, such as:

$$
\max _{\Omega} M-z_{4} \leqslant 0
$$

Determining $z_{4}$ is one of the major issues of the sizing phase.

\section{Fuel system}

The capacity of the fuel system, $z_{5}$, yields the constraint

$$
\max _{\Omega} M_{\text {fuel }}-z_{5} \leqslant 0 .
$$

The maximum fuel weight and the maximum gross weight differ from the previous limitations owing to the fact that they can be considered as global variables. While previous limitations are indeed assessed at each time step of the mission, these ones are linked to the global outputs of the mission: the take-off weight and the required fuel weight.

\section{Engine available power}

Engine can only provide a specific amount of power that depends on the flight condition. The maximum available power, $z_{6}$, is defined at sea level and at a temperature of $15^{\circ} \mathrm{C}$. This limitation can be transposed in any flight conditions using the following equation:

$$
W e=k_{\mathrm{e}} z_{6}\left(\frac{\rho}{1.225}\right)^{k_{\mathrm{e}}^{\prime}}\left(1-k_{\mathrm{e}}^{\prime \prime}(T-288.15)\right)
$$

with $k_{\mathrm{e}}, k_{\mathrm{e}}^{\prime}$ and $k_{\mathrm{e}}^{\prime \prime}$ positive real coefficients.

The engine limitation leads to the following equation:

$$
\min _{\Omega}\left(W_{r q}-W_{e}\right) \leqslant 0
$$

As described, all previous limitations have their specificity. They have been selected as a representative sample of the limitations encountered during a real sizing phase. An aircraft that meets each of these limitations will be referred to as feasible or sized.

\subsection{Limitation solving}

The standard sizing process consists in solving all the constraints in a separate sizing loop as presented in Figure 1.
Because the systems weights obviously increase with the limit values, minimizing them will tend to minimize one of the objectives: the empty weight. Four of the five proposed limitations entail a limit value and, in all of these cases, replacing the corresponding inequality by an equality amounts to minimizing the limit value, hence the empty weight.

However it is not the case for the rotor limitation. The available parameters are the design variables $c, R$ and $\omega$. The blade number, $b$, is linked to complex vibration and cost considerations and, as such, is not considered as a variable. Radius and rotation speed have a great impact respectively on induced power and drive system weight and, as such, should remain design variables. The chord $c$ appears to be the only parameter that can be used to solve this limitation. It should satisfy the following equality:

$$
c=\frac{5}{b R^{3} \omega^{2} C z_{\max }} \max _{\Omega} \frac{F_{n}}{\rho}
$$

\subsection{Integration into an optimization loop}

The optimization problem under consideration reads:

$$
\left(P_{1}\right) \mid \begin{array}{ll}
\text { Minimize } & F(X)=\left(f_{1}(X), f_{2}(X)\right) \\
\text { s.t } & L \leqslant X \leqslant U
\end{array}
$$

Here, $f_{1}$ and $f_{2}$ are the two objective functions, s.t. is the standard abbreviation for subject to and $L$ and $U$ are respectively the lower and upper bound of the design variables $X$. As shown in Figure 1, the limitation variables $Z$ do not appear at the optimization level. Problem $\left(P_{1}\right)$ is a bi-objective bound constrained optimization problem.

Problem $\left(P_{1}\right)$ has two objective functions, which may be conflicting: optimizing one of them may deteriorate the other one. As a consequence, we obtain a complete set of compromises between those objectives instead of a single solution. In order to rank solutions, the Pareto optimality is used [17]. Considering $u$ and $v$ two elements of $E, u$ dominates $v$ if and only if the following two conditions are satisfied:

$$
\begin{array}{r}
\forall i \in \llbracket 1 ; s \rrbracket f_{i}(u) \leqslant f_{i}(v) \\
\exists i \in \llbracket 1 ; s \rrbracket \mid f_{i}(u)<f_{i}(v)
\end{array}
$$

The set of non-dominated elements of $E$ is called the Pareto front. The aim of the multi-objective optimiser is then to converge toward this Pareto front.

Our problem turns out to be non-convex so none of the objective aggregation methods (see e.g. [17]) can be used. Moreover, some elements of the global model are issued from black boxes or imply a local numerical solving. Gradient-based methods are then not applicable. Besides, this code is designed for industrial applications; robustness is therefore an important issue. For all these reasons, meta-heuristics and more precisely genetic algorithms [18] appear to be the best candidates. The Matlab global optimization toolbox MOGA (Multi-Objective Genetic Algorithm) [19] has been used to generate the results 
presented in Section 4.1. It is a standard genetic algorithm adapted to multi-objective optimization using the Pareto rank as the fitness function.

For the purpose of this study, the mutation and crossover fractions [19] have been set to 0.1 and 0.8 , respectively. The selection of the element of the population accessing to the crossover stage is done through the tournament method [19] implying a couple of elements of the population.

The first test case is a direct transcription of the standard predesign approach $[2,3]$. It does not account for any adaptation of the sizing constraint solving but only involves modifications of the rotorcraft modelling. The results show that the sizing loop requires an average of 7 iterations to converge.

The second strategy proposes to take advantage of the presence of the genetic optimizer to solve the non-linear constraints [20]. Since no sizing iteration will be required anymore, solving the limitations within the genetic algorithm will divide the computation time required for one element of the population by a factor of 7 . It also results in an increase of the search space dimension. However, it is generally advised in the literature to choose a population size proportional to the size of the bit string representing the design variables [21]. Because the addition of the limit values as design variables only doubles this size for the considered test case, a gain of computation time of a factor of three can be expected for the evaluation of a single population. The problem considered becomes the following:

$$
\left(P_{2}\right) \mid \begin{aligned}
& \text { Minimize } H\left(X^{\prime}\right) \\
& \text { s.t } L \leqslant X^{\prime} \leqslant U
\end{aligned}
$$

where $X^{\prime}$ is an element of $E \times K$ and $H$ is the penalized objective function. Penalizations based on fixed multiplicative factors or fixed values have been tried. They however did not provide any satisfactory results. The penalization function proposed has been selected because it quantifies the distance to a correctly sized rotorcraft. As such, it helps solving the limitation constraints. The function can be expressed as follow:

$$
H\left(X^{\prime}\right)=\left\{\begin{array}{cc}
\left(D_{1}, D_{2}\right)+D_{3} \cdot\left\|g\left(X^{\prime}\right)\right\| & \text { if } g\left(X^{\prime}\right) \leqslant 0 \\
\left(f_{1}\left(X^{\prime}\right), f_{2}\left(X^{\prime}\right)\right) & \text { otherwise }
\end{array}\right.
$$

where $D_{1}$ and $D_{2}$ are real values selected in order to ensure that unfeasible rotorcrafts cannot have better objective values than feasible ones.

The results obtained with this approach are presented in Section 4.2. This Pareto front does not extend to the region covered by the one obtained previously and cannot be considered satisfactory. A third approach has then been tested: a hybrid of the previous ones. The aim is to gather the advantages of both. A first assessment of the limitation is done. Only the elements that do not meet these constraints are resized. The results of this approach are presented in Section 4.3.

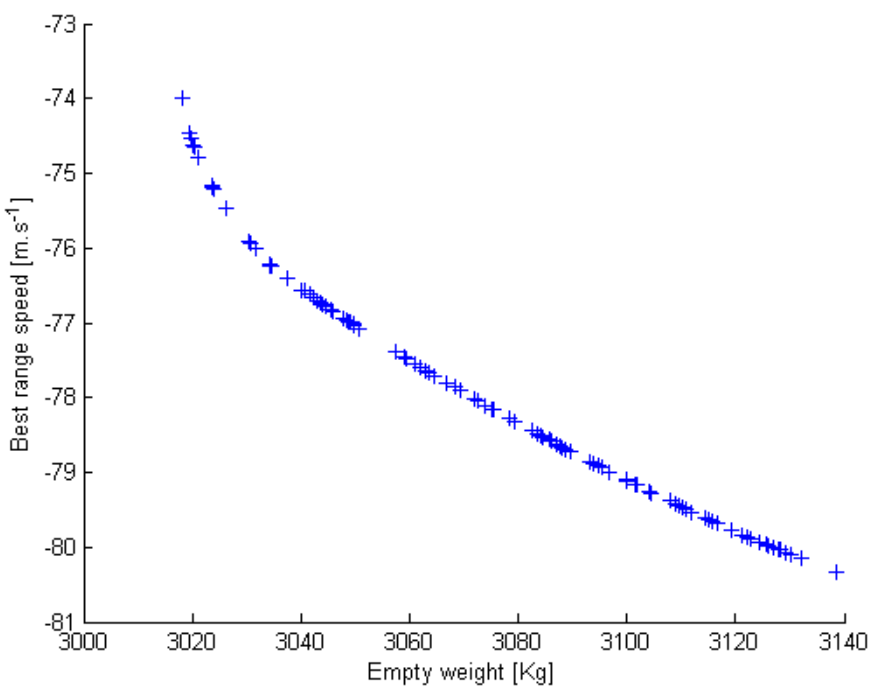

Fig. 7. Pareto obtained front with the standard approach.

\section{Results and interpretation}

The chosen test case is based on the development of a medium helicopter of the 5 ton class. The tested flight conditions are composed of three flight cases:

- Hover at $80 \%$ of its MTOW, out of ground effect at $1500 \mathrm{~m}$ in ISA+20 conditions.

- Hover with 8 passengers, 2 crew members and the maximum fuel weight at sea level standard conditions.

- Cruise at the best range speed at the maximum gross weight at $1000 \mathrm{~m}$ in standard conditions.

and one mission:

- Flight a standard Oil and Gas mission carrying 6 passengers at $300 \mathrm{NM}$.

The helicopters are defined by six design variables and six limit values. They will be optimized according to both the empty weight and the best range speed found in the third flight case.

Several population sizes have been tested for each of the three proposed strategies. Results are presented only for the population size that provides the best convergence time. The number of objective function evaluations is also provided as a representative of the computation time.

\subsection{Direct application to actual sizing methods}

As presented, the first approach is directly based on the standard predesign approach. All the limitations are calculated in an independent sizing loop as described in Figure 1. The best convergence time has been reached for a population size set to 100 . It took an average of 110 generations to obtain a complete convergence and an average of 80000 function evaluations.

The obtained Pareto front is presented in Figure 7 below. 


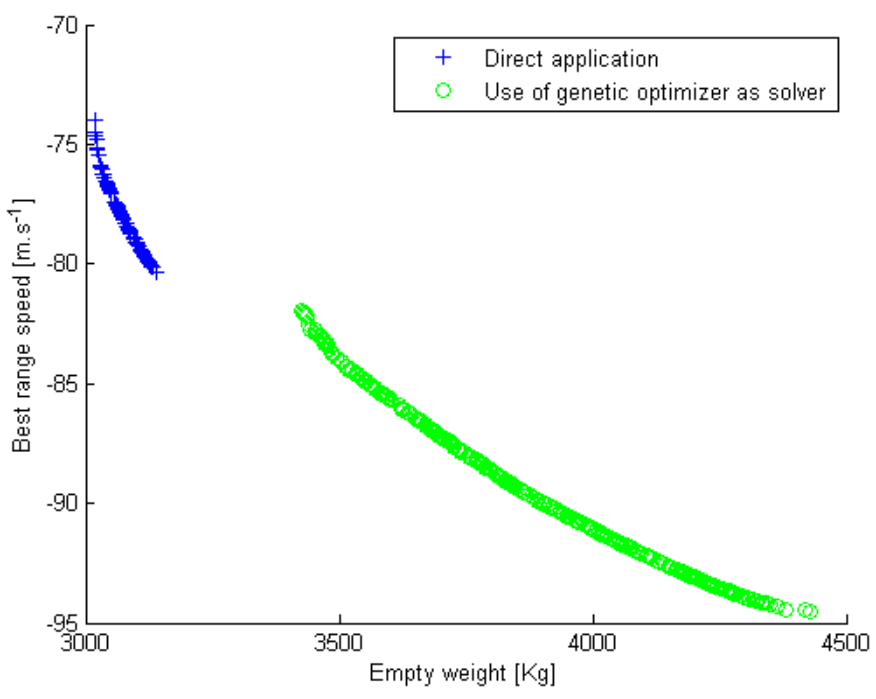

Fig. 8. Pareto front obtained with the genetic solving.

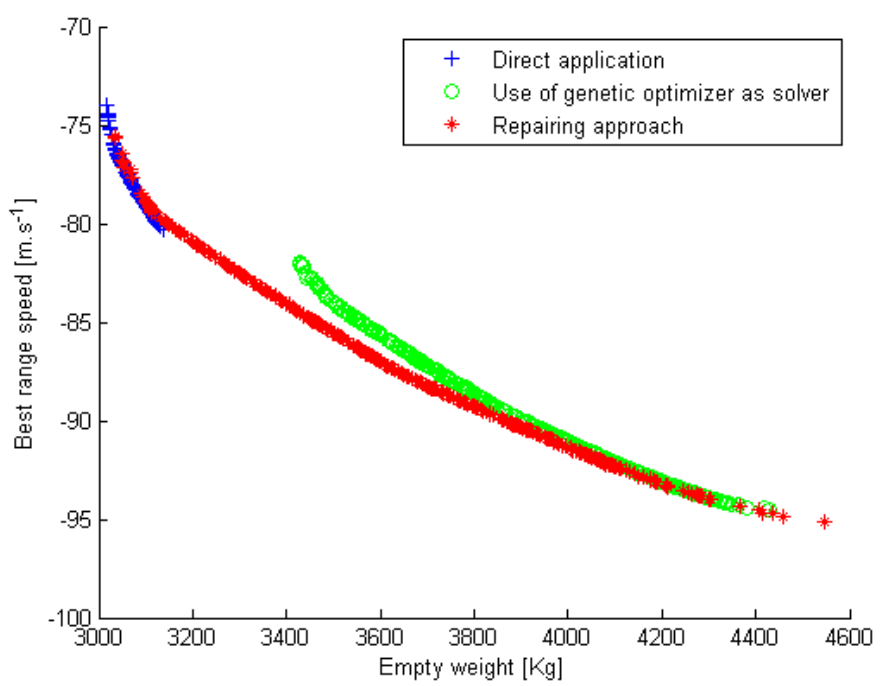

Fig. 9. Pareto front obtained with the repairing approach.

models. The standard sizing convergence approach has been proven inefficient in the frame on a multi-objective optimization. Two different approaches have been proposed and compared.

In the proposed formulation we defined limit value variables and the associated limitation functions. This formulation allows converting the standard unconstrained optimization of sized aircraft into a constrained optimization that performs both sizing and optimization at the same level. This approach provides a much better spread of the solution within the Pareto front and a convergence computation time divided by a factor of 2.6. However this approach leads to a loss of fidelity at the Pareto front ends. A hybrid formulation has then been proposed. It applies a repairing function to unfeasible rotorcrafts and, as such, helps convergence towards the reduction of empty weight. This approach gathers the advantages of the previous ones. It indeed produces an extended Pareto front with a number of function evaluations twice smaller than for the standard approach.

\section{References}

[1] D P. Raymer, Enhancing aircraft conceptual design using multidisciplinary optimisation, 2002

[2] P.M. Basset, A. Tremolet, F. Cuzieux, C. Schulte, D. Tristant, T. Lefebvre, G. Reboul, M. Costs, F. Richez, S. Burguburu, D. Petot, B. Paluch, The C.R.E.A.T.I.O.N project for rotorcraft concepts evaluation : The first steps, ID 207, 37th European Rotorcraft Forum, 2011

[3] P.M Basset, A. Tremolet, F. Cuzieux, G. Reboul, M. Costes, D. Tristant, D. Petot, C.R.E.A.T.I.O.N the Onera multi-level rotor craft concepts evaluation tool: the foundations, Future Vertical Lift Aircraft Design Conference, 2012

[4] J.M.G.F. Stevens, J.F. Boer, W.F. Lammen, W.J. Vankan, C. Sevin, Helicopter pre-design strategy: design to mass or design to cost? Advances in Collaborative Civil

An integrated sizing model has been proposed. It in-
cludes both new performances and weight assessment 
Aeronautical Multidisciplinary Design Optimization, AIAA, 2010

[5] W. Johnson, NDARC-NASA Design and Analysis of Rotorcraft theorical basis and architecture, 2010

[6] S. Sartorius, A tool for rotorcraft pre-design sizing, American Helicopter Society 67th Annual Forum, 2011

[7] W. Johnson, Helicopter Theory, Dover Publications, 2012

[8] R.W. Prouty, Helicopter Performance, Stability, and Control, Chapter Performance analysis, R.E. Krieger Publishing Company, 2003

[9] Experimental study of main rotor/tail rotor/airframe interactions in hover, Text and figures, Sikorsky Aicraft Division, 1983, Vol. 1

[10] S.F. Hoerner, Fluid-Dynamic Drag: Theoretical, Experimental and Statistical Information

[11] J.G. Leishman, Principles of Helicopter aerodynamics, Reynolds and Mach effect, pp. 350-360

[12] Polge, Calcul pratique des performances d'hélicoptère, CAL01012, Sud Aviation, 1965

[13] A.T. Bellocchio, Drive system design methodology for a single main rotor heavy lift helicopter, Georgia Institute of Technology, 2005
[14] J.A. Crabtree, Society of Allied Weight Engineers, Inc. 17th National Conference, Technical Paper No. 177, Weight Estimation for Helicopter Design Analysis, May 1958

[15] P. Lefort, J. Hamann, L'Hélicoptère - Théorie et Pratique, $9^{\text {ème }}$ édition, Chiron-Paris, 1999

[16] R.W. Prouty, Helicopter Performance, Stability, and Control, Weight estimates and Balancing, R.E. Krieger Publishing Company, 2003, pp. 662-668

[17] Y. Collette, P. Siarry, Multiobjective Optimization: Principles and Case Studies, Springer, 2003

[18] I. Boussaïda, J. Lepagnot, P. Siarry, A survey on optimization metaheuristics, Information Sciences, 237 (2013) 82-117

[19] D. Kalyanmoy, Multi-Objective Optimization Using Evolutionary Algorithms, John Wiley \& Sons, 2001.

[20] I.M.M. El-Emary, M.M. Abd El-Kareem, Towards Using Genetic Algorithm for Solving Nonlinear Equation Systems, World Appl. Sci. J. 5 (2008) 282-289

[21] E.G. Talbi, Metaheuristics: from design to implementation, Wiley, 2005 